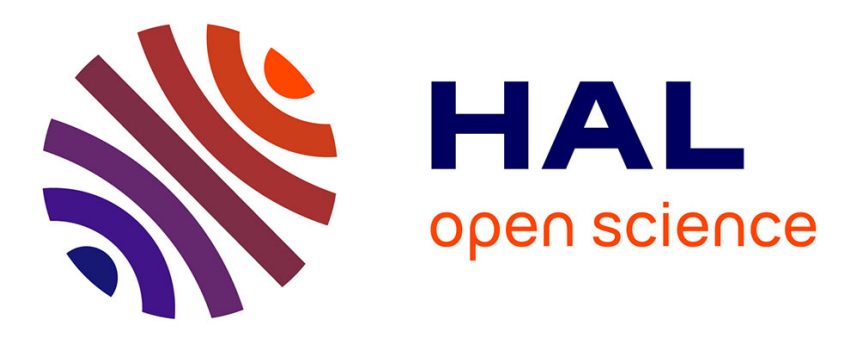

\title{
Polymer resonators sensors for detection of sphingolipid gel/fluid phase transition and melting temperature measurement
}

\author{
Qingyue Li, Véronique Vié, Hervé Lhermite, Etienne Gaviot, Claire
}

Bourlieu-Lacanal, Alain Moréac, Denis Morineau, Didier Dupont, Sylvie

Beaufils, Bruno Bêche

\section{To cite this version:}

Qingyue Li, Véronique Vié, Hervé Lhermite, Etienne Gaviot, Claire Bourlieu-Lacanal, et al.. Polymer resonators sensors for detection of sphingolipid gel/fluid phase transition and melting temperature measurement. Sensors and Actuators A: Physical, 2017, 263, pp.707-717. 10.1016/j.sna.2017.07.037 . hal-01572203

\section{HAL Id: hal-01572203 \\ https://hal.science/hal-01572203}

Submitted on 8 Aug 2017

HAL is a multi-disciplinary open access archive for the deposit and dissemination of scientific research documents, whether they are published or not. The documents may come from teaching and research institutions in France or abroad, or from public or private research centers.
L'archive ouverte pluridisciplinaire HAL, est destinée au dépôt et à la diffusion de documents scientifiques de niveau recherche, publiés ou non, émanant des établissements d'enseignement et de recherche français ou étrangers, des laboratoires publics ou privés.

\section{다(1)(2)}

Distributed under a Creative Commons Attribution - ShareAlikel 4.0 International 


\title{
Polymer Resonators Sensors for Detection of Sphingolipid Gel/Fluid Phase Transition and Melting Temperature Measurement
}

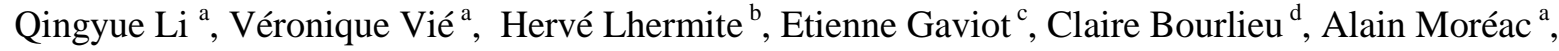 \\ Denis Morineau ${ }^{a}$, Didier Dupont ${ }^{\mathrm{e}}$, Sylvie Beaufils ${ }^{\mathrm{a}}$, Bruno Bêche ${ }^{\mathrm{a},{ }^{*}}$ \\ ${ }^{a}$ Institut de Physique de Rennes, IPR CNRS 6251, Université de Rennes 1, 35042 Rennes, France \\ b Institut d'Electronique et de Télécommunications de Rennes, IETR CNRS 6164, Université de Rennes 1, \\ 35042 Rennes, France \\ ${ }^{\mathrm{c}}$ Laboratoire d'Acoustique de l'Université du Maine, LAUM CNRS 6613, Université du Maine, 72000 Le \\ Mans, France \\ d Ingénierie des Agropolymères et Technologies Émergentes, IATE UMR 1208, Université de Montpellier, \\ France \\ ${ }^{\mathrm{e}}$ Science et Technologie du Lait et de l'Oeuf, STLO INRA 1253, Agrocampus Ouest, 35042 Rennes, France \\ * Corresponding Authors at : Institut de Physique de Rennes, IPR CNRS 6251, Université de Rennes 1, 35042 \\ Rennes, France \\ E-mail address: bruno.beche@univ-rennes1.fr (B. Bêche)
}

\begin{abstract}
This work describes a low-cost biophotonic sensor shaped by way of cheap processes as hybrid silicon/silica/polymer resonators able to detect biological molecule gel/fluid phase transition as lipids at very low concentration (sphingomyelin). The photonic structure is composed of specific amplified deep UV photoresist-polymer waveguides coupled by a sub-wavelength gap with racetrack microresonators allowing a low temperature-dependent operation ranging from 16 to $42{ }^{\circ} \mathrm{C}$. The temperature dependent wavelength shift and the thermo-optic coefficient characterizing the quantified resonances and opto-geometric properties of the device have been evaluated, highlighting an enough low thermal features of the whole system for such application. With an appropriate vesicle lipid deposition process specific in biology associated to an apt experimental bio-thermo-photonic protocol (made of serial optical resonance spectra acquisitions with statistical treatments), the dynamic evolution of the sphingomyelin lipid phase transition was assessed: then, the ability to detect their own gel/fluid transition phase and melting temperature has been demonstrated with a mass product factor $10^{7}$ lower than that of more conventional methods. The equilibrium of the regime of the resonators was highlighted as being broken by the dynamic of the sphingomyelin and its own phase transition prior relevant detection.
\end{abstract}

Keywords: Integrated sensors, photonics, resonators, deep UV polymer, gel/fluid phase transition, sphingomyelin lipids 


\section{Introduction}

Optical microcavities or microresonators have been most generic components, for the last decade, so as to design and fabricate integrated photonic devices leading to the development of numerous applications in science. They include as much fundamental physics studies as the researches for engineering telecommunications, biophysics, biochemical and biology [1-3]. Indeed, optical microcavities are quite welcome devices allowing to control optical fields as regards their spatial localization and very lifetime. To a certain extent, as such resonant quantifications met in physics are due to a geometric recirculation of the light (called whispering gallery modes - WGMs), they increase both the field and merit of integrated photonics regarding a significant set of optical telecommunications versatile applications (including filters, switches, modulators, de/multiplexing components, lasers...) together with sensors for metrology with platform analysis and relevant detection procedure. The spatial localization of the light, due to the fundamental optical laws and the nature of the evanescent part of the light which is not completely confined, acts as a tunable probe for the surrounding and enables the light to interact and feel the environment. For example, considering the recent development of telecommunication components, specific low thermal properties have been widely searched so as to obtain a response function or a signal transduction of operative devices as much as possible independent in temperature [4]. Such significant and crucial properties allow a striking improvement of the components operating range with enhanced stability in strong environmental conditions. Regarding sensors applications requiring such drift-less properties, the response signal of ideal components should be quasi temperature independent, allowing then output signals only depending on the samples to be detected. Sundry approaches and processes have been developed on successive classes of materials in order to shape, on 2D planar technologies or 3D hybrid configurations, different architectures of microresonators (ring, disk, stadium, racetrack, toroid, cylinder, sphere and so on) [5-9]. In an overall landscape of technologies, resonators based on organic materials exhibit a lot of advantages due to the well-established printing or photolithographic technologies, the versatility of the polymer properties and functionalization and the possibility to be shaped starting from a liquid photoresist material with fluidic thin layer specific deposition principles [9-13]. All these are reproducible ways and concepts. Globally, the reasons of the progress in this research field are various and easily justified with the potential of numerous available materials, the simplicity of the relevant processes, the specific methods and measurements protocols and low costs of the entailed devices. Furthermore, together with designs immune against temperature changes, one can expect the ability to detect a specific function for sensors applications, an apt recycling or the possibility of further uses of the components in biodetection after simple water washing, the stability of the optical response functions and so on. More recently, after the substantial development in telecommunications, organic materials based resonators have received intensive attention in metrology and sensing environmental applications, biology, medical and healthcare diagnostics, food quality control and security. Such photonics sensors relying on resonators have become the subject of comprehensive research with sizeable developments of enhanced sensing platforms devoted to the label-free detection of a wide variety of chemical and biochemical [14-18], biological agents and biomedical materials [19-28].

Sphingomyelin (SPH) is a type of sphingolipid that can also be classified as sphingophospholipids, found in animal cell membranes. SPH is especially prominent in myelin, a membranous sheath that surrounds and insulates the axons of numerous neurons [29-32]. In humans, SPH represents $85 \%$ of all sphingolipids, and typically make up 10-20 mol \% of plasma membrane lipids with higher concentrations found in nerve tissues, red blood cells, and the ocular lenses... Such a plasma membrane component participates in many signaling pathways. The metabolism of SPH creates many products that play significant roles in the cell. As an example SPH undergoes significant interactions 
with cholesterol. The latter has the ability to strongly shift the liquid to solid phase transition in phospholipids. Since sphingomyelin transition temperature stands within physiological temperature ranges, cholesterol can play a significant role in the phase of sphingomyelin.

The present work is aimed at exploring and investigating low-cost and easily reproducible polymeric biophotonic sensors integrated on a chip-device which features an enough low thermal behavior so as to detect SPH phase transition. They are devoted to perform efficient sphingomyelin lipid first order phase transition detection based on a gel-liquid state-change and then melting temperature determination. The lipids used, here, is the sphingomyelin (SPH), which plays a crucial role in the biology and the biochemical aspect in the particular function of plasma membrane of cells. The first section describes the global fabrication involving thin layer processes, starting from the chemical and materials aspects to achieve suitable deposition of biological samples, which are relevant in our compound approach. This comprises the use of attractive organic amplified photoresins with specific deep UV technology associated with an appropriate lipid deposition process related to biology. In addition, sundry characterization methods regarding the on-chip-device are described so as to validate both its concept and design. Furthermore, we also detail in a second section, the versatile principle of operation and justify the measurements approach together with experiments developed for such a specific biometrology ascribed to temperature dependent lipid phase transition detection. The appropriate bio-thermo-photonic method and protocol, based on serial optical resonance spectra acquisitions and statistical treatments on a sensing platform design, are also depicted. An adequate low thermal feature of the chip device (sensors without lipid deposit) is established: the system is not so sensitive to temperature changes in a wide range from $16^{\circ} \mathrm{C}$ to $42^{\circ} \mathrm{C}$ and then is appropriate to detect specific molecules localized on the top of the chip so as to assess their relevant biomechanisms. Then in the third and last section, the ability to follow the dynamic evolution of sphingomyelin (SPH) with temperature by detecting their own gel/fluid transition phase can be emphasized, together with the determination of the melting temperature due to changes of the specific parameters accounting for the optical spectra. The ability to detect their own gel/fluid transition phase and melting temperature will be clearly demonstrated with a mass product factor $10^{7}$ lower than that of differential scanning calorimetry method in comparison.

\section{Design, materials and processes, realization and characterization of the structure}

\subsection{Theory on opto-geometric considerations concerning the optical single-mode behavior}

So as to operate on quasi- $\mathrm{TE}_{00}$ and $-\mathrm{TM}_{00}$ single-mode eigenvectors, adequate and straightforward simulations have been previously achieved considering the theory of electromagnetism in waveguides allowing us to obtain eigenvalues equations: then, all the series of quantified effective propagation constants $\beta=\mathrm{k}_{0} \cdot \mathrm{n}_{\text {eff }}$ or effective indices $\mathrm{n}_{\text {eff }}$ can be settled defining then the apt opto-geometric parameters for such photonic structures. These typical dimensions (h-height and w-width) regarding refractive indices enable us to operate with exclusive monomode $\mathrm{TE}_{00}-\mathrm{TM}_{00}$ optical modes. The methodology supporting such simulations consists in solving the J.C. Maxwell's equations in each part of the whole system while taking into account the continuity properties of the electromagnetic fields so as to obtain the so-called eigenvalues equations that highlight directly the overall quantifications of the fields viewed as eigenvectors. Such photonic structures or opto-geometrical systems show-off an intrinsic asymmetry especially in the optical indices entailing a cut to occur in the dispersion curves of the modes. As an example, considering the first operation of quantification along the direction perpendicular to the wafer surface with regard to the apt electromagnetism theory, it is easy to define the cut-thickness notion regarding both the $\mathrm{TE}_{\mathrm{m}}$ and $\mathrm{TM}_{\mathrm{m}}$ modes (m integer) within our structure: 


$$
\mathrm{h}_{\mathrm{cut}}=\frac{\lambda_{0}}{2 \pi\left(\mathrm{n}_{\mathrm{DUV} 210}^{2}-\mathrm{n}_{\mathrm{SiO2} 2}^{2}\right)^{1 / 2}}\left\{\operatorname{arctg}\left[\eta_{\text {DUV210/upper-cladd }}\left(\frac{\mathrm{n}_{\mathrm{SiO} 2}^{2}-\mathrm{n}_{\text {upper-cladd }}^{2}}{\mathrm{n}_{\mathrm{DUV} 210}^{2}-\mathrm{n}_{\text {SiO2 }}^{2}}\right)^{1 / 2}\right]+\mathrm{m} \pi\right\},
$$

Here, $\mathrm{n}_{\text {material }}$ stands for the relevant refractive indices, considering the DUV210 polymer core and respective claddings (lower $\mathrm{SiO}_{2}$, upper air or lipids) at $\lambda_{0}$-wavelength; also, $\eta_{\text {DUV210/upper-cladd }}$ $=1$ or $\left(\frac{n_{\text {Duv210 }}}{n_{\text {upper-cladd }}}\right)^{2}$ for their respective $\mathrm{TE}_{\mathrm{m}}$ and $\mathrm{TM}_{\mathrm{m}}$ polarizations. Below the $\mathrm{h}_{\mathrm{c}}$-values at $\mathrm{m}=0$, with no eigenvectors allowing the light to occur, a consequent forbidden area is located out of the cone of light ; the latter gives rise to the dispersion curves related with the family of bounded or guided modes. Between the ranging of $\mathrm{h}_{\mathrm{c}}$-values (Table 1) corresponding to the cut-thicknesses at respectively $[\mathrm{m}=0 ; \mathrm{m}=1]$, both $\mathrm{TE}_{0^{-}}$and $\mathrm{TM}_{0}$ single-modes occur and propagate with a high probability of presence. In conclusion, a rib waveguide typically $800 \mathrm{~nm}$ in thickness $\times 2 \mu \mathrm{m}$ DUV210, arranged onto $\mathrm{SiO}_{2} / \mathrm{Si}$ (shaped as described previously into the paper) makes certain to maintain a single-mode characteristic: then, the device may be operated with respectively the optical propagation (straight or bent into the micro-resonators), an apt optical coupling between the waveguide and the microresonator element featuring the optical resonance phenomenon.

\subsection{Thin layer processes, microtechnology}

On a silicon substrate, a thermal silica layer is formed by wet oxidation method. We must first clean the wafer with the method RCA (developed by 'Radio Company of America'), to avoid all impurities. The plate is then put in a quartz oven with torch, the gas $\mathrm{H}_{2}$ and oxidation gas $\mathrm{O}_{2}$ being located in the flame for the combustion. A 3 hours stage in the oven at $1075{ }^{\circ} \mathrm{C}$ can form $1000 \mathrm{~nm}$ of silica on the surface of the wafer, creating the silica lower cladding. After oxidation, the annealing at $700^{\circ} \mathrm{C}-800^{\circ} \mathrm{C}$ strengthens the single crystal structure. Such a thickness guarantees a highly stable and homogeneous refractive index under the organic waveguides and micro-resonators (MRs) so as to decrease the optical radiation losses regarding the propagation modes. An important key point refers to the use of a polymer called UV210 from the photoresist chemistry (Shipley) based on deep UV processes and light/matter interactions. We develop such a deep UV lithography approach at $248 \mathrm{~nm}$ (that is not the conventional peak, called i-line at $365 \mathrm{~nm}$ ) by way of coupling a straightforward mercury short arc lamp (HBO 1000W/D, OSRAM) with an adequate and selective filter, as a significantly low cost method compared with electron beam lithography and $\mathrm{ArF}$ or $\mathrm{KrF}$ excimer laser or optical systems using $F_{2}$. Such an organic exposure to deep UV radiation at $\lambda_{\text {flash }}=248 \mathrm{~nm}$ enables valuable low-cost realizations of sub-wavelength photonics structures considering the gap between waveguides and micro-resonators (MRs) within the order of $\lambda_{\text {flash }}$ (due to the Raleigh's criteria and its associated spatial resolution limit). Such a deep UV (DUV) polymer made of poly p-hydroxystyrene and poly t-butyl acrylate is called an amplified chemical photoresist including a photo acid generator (PAG) to increase the sensitivity with UV energy exposure as regards such a specific photolithography mechanism. Indeed the DUV-insolation induces the production with the PAG of a small quantity of acid acting as a catalyst during the exposure. Thermally activating this acid with a post exposure bake causes the proton $\mathrm{H}+$ to act as a catalyst and unblock the group from the PHS. Then, the cascade of acid-activated chemical changes brings about a change in polarity of the polymer which goes from lipophilic to hydrophilic states. This make the PHS soluble in the exposed areas. Thus, DUV exposed areas become soluble in a basic developer as the tetra-methyl ammonium hydroxide (Microposit MF CD-26). Such immersion in the developer allows us to get the whole chip featuring the MRs. In order to optimize the test and the adequate photonic injection, integrated chips on such DUV210/ $\mathrm{SiO}_{2} / \mathrm{Si}$ 
multilayers are cleanly cleaved by way of a diamond tip. The adequate and overall optimized processes are illustrated in Table 2.

\subsection{Characterizations of the structures, atomic force microscopy, micro-Raman spectroscopy and imaging}

Strict quality controls of the chip (materials, properties, geometries, sub-micron dimensions, surface aspects and so on) are then necessary with the help of various technologies and instrumentations concerning imaging and analysis: optical microscopy that may involve imaging with Nomarski operation in Differential Interference Contrast (DIC), Atomic Force Microscopy (AFM, BRUCKER Nanoscope 8), Scanning Electron Microscopy (SEM) and Raman micro-spectroscopy and imaging (Fig. 1). Top view photographs by optical imaging and AFM allow to measure either globally or specifically located in specific areas, various geometric parameters regarding the waveguide/racetrack MRs devices. Then, may be assessed: the g-gap of the evanescent physics of the coupling or the optical tunnel effect equal to $540 \mathrm{~nm}$ (for an interesting selectivity), the coupling length $\left(\mathrm{L}_{\mathrm{c}}=15 \mu \mathrm{m}\right)$ allowing to the light the time both to transit and couple into the resonant part of the MR circuit, plus the respective dimensions of radius $\mathrm{R}=15 \mu \mathrm{m}, \mathrm{w}=2 \mu \mathrm{m}$ and $\mathrm{h}=860 \mathrm{~nm}$ allowing only the single-mode existence of mathematic eigenvectors $\left(\mathrm{TE}_{00}\right.$ and $\left.\mathrm{TM}_{00}\right)$ considered with a $\lambda_{0}=795 \mathrm{~nm}$ propagation wavelength. Moreover, the DUV 210 surface analysis with AFM has confirmed a roughness lower than $2 \mathrm{~nm}$ clearly minimizing the optical losses due to parasitic diffraction. The photonic structure was imaged with the MultiMode 8 , an atomic force microscope from Brucker. The images were acquired in tapping mode in air. The z-scale is $1 \mu \mathrm{m}$ and the image size is $(80 \times 80) \mu \mathrm{m}^{2}$. The studies by micro-Raman vibrational molecular spectroscopy and analysis measurements without destruction of materials, were carried out and results recorded on a LabRAM HR 800 (Horiba Scientific, Jobin-Yvon) fitted with a high resolution spectrometer with confocal microscopy system and combined with a $633 \mathrm{~nm}$ He-Ne laser used in visible configuration and excitation. The LabRAM HR800 Raman Spectrometer (from Horiba Scientific company, Jobin-Yvon) is a high-resolution spectral spectrometer coupled with a confocal microscope, several laser sources (633 nm He-Ne), (785 $\mathrm{nm}$ Toptica) and (532 nm Coherent) and nano-positioners. The coupling with a confocal microscope enables us to imaging a sample in $2 \mathrm{D}$ with a spatial resolution of the order of the spot size $(0.9 \mu \mathrm{m})$ in the focal plane and a spectral resolution of typically $1 \mathrm{~cm}^{-1}$ per pixel. An apt laser excitation power lower than $0.1 \mathrm{~mW}$ is devoid of heating effect onto the photonics chip. Due to the 2D-planar geometry of the sensor, Raman spectroscopy is performed assuming a two dimensional analysis. Fig. 1(c) and 1(d) represent respectively the micro-Raman spectroscopy analyses and detection of various signal signatures of each material along the cleaved face in particular $\mathrm{Si}$ (peaks positions at $521 \mathrm{~cm}^{-1}$ and its quasi-doubled value due to the second harmonic coupling), and the specific complex signal of the organic DUV210 for example with its stretching mode of phenyl ring (s.m. CC) at $1002 \mathrm{~cm}^{-1}$ and the bending mode of aromatic olefinic (b.m. CCH) at $855 \mathrm{~nm}^{-1}$ [33]. Furthermore, filtering the enlarged signature (Fig. 1(d)) and then choosing specific peaks characterizing the DUV210 (for example at 1002 and $521 \mathrm{~cm}^{-1}$ ) it is possible to image on 2D or 1D the waveguide/MR structure for a stringent quality control on geometry and materials: are also enabled measurements of specific features crucial for such photonic devices and principles (MR aspect, g-area coupling, prospective structure defaults and DUV210 material properties). This whole set of analyses and methods entails a cogent validation of the above-mentioned serial thin layers processes with a view to undertaking mass production. 


\subsection{Lipids, fusion vesicle deposition method}

Concerning the lipid substance and deposition, Fig. 2(a) and 2(b) represent respectively the chemical structure of the sphingomyelin and the schematic diagram of the vesicle fusion deposition. Milk sphingomyelin (MSM) was purchased from Avanti Polar Lipids (Alabaster, AL, U.S.A.) under lyophilized powder form with the purity of $99 \%$. After solubilization in chloroform/methanol (1:2, $\mathrm{wt}$ ), the solvent is removed under nitrogen flux. Then, the lipid film was solubilized in Milli-Q water at the final concentration of $1 \mathrm{mg} / \mathrm{mL}$. This aqueous solution was sonicated until it appears clear in order to form a suspension of small unilamellar vesicles. The temperature was maintained over $50^{\circ} \mathrm{C}$ to keep the lipid in fluid phase. A droplet of $3 \mu \mathrm{L}$ of the lipid solution was deposited on the top of the device, amounting to $\mathrm{m}_{\mathrm{MSM}}=3 \mu \mathrm{g}$ with such a solution at $1 \mathrm{mg} / \mathrm{mL}$. The sedimentation and vesicle fusion lead to a multilayer formation on the top of the device. Then, a very low flux was applied to evaporate the water. The surface covered with the lipid film is about $5.5 \mathrm{~mm}^{2}$. Thanks to compression isotherms performed on the Langmuir trough, the mean molecular area of lipids can be determined, and for MSM this value is around $70 \AA^{2}$. The number of molecules necessary to cover homogeneously the stain of $5.5 \mathrm{~mm}^{2}$ with one layer is $7.86 \times 10^{12}$ molecules (as the ratio of the two previous surfaces). Knowing the molar weight of MSM $\left(\mathrm{MM}_{\mathrm{MSM}}=801.22 \mathrm{~g} / \mathrm{mol}\right)$, and with $\mathrm{N}_{\mathrm{A}}=6.022 \times 10^{23} / \mathrm{mol}$ the Avogadro number, the number of molecules deposited is then $\left(\mathrm{m}_{\mathrm{MSM}} / \mathrm{MM}_{\mathrm{MSM}} \times \mathrm{N}_{\mathrm{A}}\right)=2.25 \times 10^{15}$, inducing the arrangement of about 300 lipid layers. The thinness is then estimated with 300 layers of lipids. The thickness of bilayers being usually given around $5 \mathrm{~nm}$, then a single layer may be estimated about $2.5 \mathrm{~nm}$ and the estimated whole thickness of the film averages $750 \mathrm{~nm}$.

\section{Method, experimental protocol and measurement experiment}

\subsection{Synoptic of the method and experimental protocol}

Fig. 3(a) highlights the synoptic of the method with the experimental protocol and principles of measurements (without or later with previous lipid deposition) starting from the photonic platform arrangement devoted to optical preparation mode, injection and operation control, until the methodology of dynamic spectral measurements and statistical treatments in time so as to detect such lipid phase transition precisely. An overall schematic diagram and presentation of the platform and materials of various controls (position, thermal environment) and detection is presented in the following. Fig. 3(b) presents typical different ways to top view imaging of the photonic device under test with cross-sectional detection and imaging of the singlemode, allowing monitoring and verifying both the monomode light propagation $\left(\mathrm{TE}_{00}\right.$ or $\left.\mathrm{TM}_{00}\right)$ with its adequate coupling from the waveguide to the resonators and the apt circulation into the racetrack loop. Fig. 3(c) describes a typical resonant spectrum (at fixed temperature) of the photonic device detected and recorded with the optical spectral analyzer (OSA Ando AQ-6315E and Anritsu MS9710C) and relevant computer, with $\lambda_{i}(i$, integer) the peaks wavelengths, $\Delta \lambda$ and $\delta \lambda$ parameters respectively associated with the resonant wavelengths, the Free Spectral Range (FSR) and the Full Width Half maximum (FWHM). The FSR can also be determined in parallel with a Fast Fourier Transform (FFT) calculus on the comb-shaped periodic wavelength allowing then its accurate quantification.

As regards temperature dependence, the resonance wavelength depends on temperature due to the thermo-optic coefficient of the materials. Sensors (or telecommunications) components operating in low-thermal mode are most highly sought-after since they exhibit a significantly low temperature drift. Then, they are suited to operate over a wide temperature range. Indeed, when devoted to the specific detection of a particular physical/chemical/biological attribute (or to the transmission of information), the detection (or transmission) abilities of such sensors, being more stable in temperature, prove 
therefore to be more accurate. Their relevant responses are only related to the expected measurands while minimizing the intrinsic thermal drift of such components. Then, at first, so as to study the temperature dependence, experiments were performed onto the global photonic platform to measure the response of the resonator to changes of temperature within the range [16-38 $]^{\circ} \mathrm{C}$. The experimental protocol is the same as the one defined previously in Fig. 3 allowing recording the various spectral analysis and the evolution of parameters function of temperature, with air as upper cladding (that is without the sphingomyelin (SPH)-lipid).

\subsection{Thermo-optic considerations and measurements without SPH}

In this process of measurements, the transmission spectra of the $\mathrm{TE}_{00}$ polarization single-mode were measured at different steady-state temperatures with high accuracy. Fig. 4(a) represents the response in temperature of the $\mathrm{Si} / \mathrm{SiO}_{2} / \mathrm{DUV} 210$ (/air) micro-resonator (MR) sensor considering specific resonant wavelengths (various $\lambda_{\mathrm{i}}$ in Fig. 3(c)) of the spectrum. Experimentally, we can calculate an appropriate linear fit of such $\lambda(\mathrm{T})$ laws for various resonant wavelengths $\lambda_{\mathrm{i}}$ as depicted in Fig. 4(a): then, the temperature-dependent wavelength shift (TDWS) as $\mathrm{d} \lambda_{\mathrm{i}} / \mathrm{dT}$, ranging between [25$30] \mathrm{pm} /{ }^{\circ} \mathrm{C}$ is quantified over the $40 \mathrm{~nm}$ spectral range (in wavelength) of the broadband laser source $\left(\lambda_{0}=795 \mathrm{~nm}\right)$ : then, a low trend of red-shift in temperature is clearly entailed. Thus, such an approach allows us to quantify the thermal sensitivity of the DUV210 polymer applied to design MRs. In the same way, Fig. 4(b) and 4(c) represent also the response in temperature of the device, but considering now the parameters FSR, FWHM, the Q-quality factor and the finesse F related to the resonance function considering the evolution in temperature. We can notice once again a low-thermal behavior for this virgin resonant architecture (air with upper cladding) in particular with the ultra-high stability of the FSR: its value remains almost constant to nearly $10^{-4} \mathrm{~nm}$ around its average value of $2.529 \mathrm{~nm}$ over the whole operational range of temperature $[16-38]^{\circ} \mathrm{C}$; as the FWHM is decreased, then both the quality factor $\mathrm{Q}=\lambda_{0} / \mathrm{FWHM}$ and the finesse $\mathrm{F}=\mathrm{FSR} / \mathrm{FWHM}$ regarding the resonances are naturally increased with temperature. So as to further investigate the shift resonance relevant with such a air/DUV210/ $/ \mathrm{SiO}_{2}$ association of materials, the thermal behavior of this device made of photonic waveguides can be evaluated by way of the following equation $[4,34,35]$ :

$$
\frac{\mathrm{d} \lambda_{\mathrm{i}}}{\mathrm{dT}}=\frac{\lambda_{\mathrm{i}}}{\mathrm{n}_{\mathrm{g}}^{\text {syst }}} \cdot\left[\frac{\mathrm{d} \mathrm{n}_{\mathrm{eff}}^{\text {syst }}}{\mathrm{dT}}+\mathrm{n}_{\mathrm{eff}}^{\text {syst }} \cdot \alpha_{\text {sub }}\right] \text { where } \mathrm{n}_{\mathrm{g}}^{\text {syst }}=\frac{\lambda_{\mathrm{i}}^{2}}{\text { P.FSR }}
$$

with $\mathrm{d} \lambda_{\mathrm{i}} / \mathrm{dT}$ the TDWS $\left(\mathrm{pm} /{ }^{\circ} \mathrm{C}\right), \lambda_{\mathrm{i}}$ the resonant wavelengths of the optical modes spectrum, $\mathrm{n}_{\mathrm{g}}^{\text {syst }}$ and $\mathrm{n}_{\text {eff }}^{\text {syst }}$ respectively the global optical group index of the whole structure and the eigenvalue of the overall system called effective phase index: FSR and $\mathrm{P}$ are respectively the free spectral range and the perimeter related to the resonance spectrum and to the micro-resonator, $\mathrm{dn}_{\mathrm{eff}}^{\text {syst }} / \mathrm{dT}$ the thermo-optic (TO) coefficient of the used polymer considering the global system and its photonic structure and $\alpha_{\text {sub }}=\alpha_{\mathrm{SiO} 2}$ the lower substrate expansion coefficient. As regards temperature, the entailed change in optical refractive index of the DUV210 polymer shaped in thin layer with MRs geometry is described by this particular TO coefficient $\mathrm{dn}_{\mathrm{eff}}^{\text {syst }} / \mathrm{dT}$. From equation (2), considering (in the literature) that $\alpha_{\mathrm{SiO} 2} \sim 1 \times 10^{-5} /{ }^{\circ} \mathrm{C}$ at a $795 \mathrm{~nm}$-wavelength, $\mathrm{P}=125 \mu \mathrm{m}$ (Fig. 1(a)) and FSR=2.529 nm (Fig. 4(b)) for the calculus of $\mathrm{n}_{\mathrm{g}}^{\text {syst }}$, and according to the previous average measurement of $\mathrm{d} \lambda_{\mathrm{i}} / \mathrm{dT}=27.5 \mathrm{pm} /{ }^{\circ} \mathrm{C}$ (Fig. $4(\mathrm{a}))$, the TO coefficient is then evaluated as $\left|\mathrm{dn}_{\text {eff }}^{\text {sys }} / \mathrm{dTT}\right|=5.4 \times 10^{-5} /{ }^{\circ} \mathrm{C}$. Then, compared to other polymers, the specific amplified chemical polymer DUV210, shaped on such architecture, presents a 
relatively quite reduced sensitivity against temperature as regards the optical index variable of phase [36-38]. We can also investigate the value of the dispersion in temperature regarding the global group index by differentiating $\mathrm{n}_{\mathrm{g}}^{\text {syst }}$ (equation (2), at MR's perimeter $\mathrm{P}$ constant) with $\left|\mathrm{dn}_{\mathrm{g}}^{\text {syst }} / \mathrm{dT}\right|=(1 /$ $\mathrm{P})\left[\left(\lambda_{\mathrm{i}}^{2} / \mathrm{FSR}^{2}\right) \cdot(\mathrm{dFSR} / \mathrm{dT})+\left(2 \lambda_{\mathrm{i}} / \mathrm{FSR}\right) \cdot\left(\mathrm{d} \lambda_{\mathrm{i}} / \mathrm{dT}\right)\right]$. Physically, since such a dispersion in temperature $\left|\mathrm{dn}_{\mathrm{g}}^{\text {syst }} / \mathrm{dT}\right|$ on the whole system group index is related to the resonant structure, its formal value points up two terms respectively function of the FSR dependence on temperature written as (dFSR/dT), standing for the resonant part of the device according to the notion of FSR, and the term $\left(\mathrm{d} \lambda_{\mathrm{i}} / \mathrm{dT}\right)$ which represents the more classical wavelength dispersion in temperature. Considering the average value defined in Fig. 4 (b) as $(\mathrm{dFSR} / \mathrm{dT})=2.5 \times 10^{-5} \mathrm{~nm} /{ }^{\circ} \mathrm{C}$, the first term can be evaluated as about $1.58 \times 10^{-5} /{ }^{\circ} \mathrm{C}$ while the second contributes with $1.38 \times 10^{-4} /{ }^{\circ} \mathrm{C}$. The compounded effect brings about $\left|\mathrm{dn}_{\mathrm{g}}^{\text {syst }} / \mathrm{dT}\right|=1.54 \times 10^{-4} /{ }^{\circ} \mathrm{C}$ for such MR structures that hinge on resonant wave packets and broadband laser sources. Then, this specific DUV210 polymer and associated processes devoted to design MR architectures show most promising prospects for integrated photonic applications with high stability over a typical range of temperature [15-40] ${ }^{\circ} \mathrm{C}$, clearly highlighted considering the quite minor FSR dispersion in temperature (dFSR/dT). The previous characterizations hold for advantageous novel standards for such devices as regard the following application and detection.

\section{Detection of Sphingolipid gel/fluid phase transition, results and discussions}

In order to further evaluate the operation and performance of such opto-thermal MR devices, assessments of phase transition with a specific sphingolipid called sphingomyelin were carried out onto a global platform described Fig. 5.

\subsection{Thermal consideration on SPH lipid}

The sphingomyelin is a special class of lipids found in the bio-membranes and is involved on the membrane heterogeneity. The latter is clearly highlighted either by way of fluorescence microscopy or with operating detergent solubilization on cells and is related to the presence of saturated chains on the lipids. The sphingomyelin used in this work, was purified from milk bovine and chosen because the presence of saturated chain is lower than other sources (brain or egg) [39]. At the room temperature, the hydrocarbon lipid chains display an all-trans configuration so-called gel phase. At a specific temperature (melting temperature or main temperature, $\mathrm{T}_{\mathrm{m}}$ ), a thermotropic phase transition occurs leading to a fluid phase, as a consequence of trans-gauche chain isomerizations. $T_{m}$ is affected by the saturation/unsaturation of the hydrocarbon chains; presence of saturated chains increases the $T_{m}$ value. After having implemented the fusion vesicle method deposition that is specific in biology so as to build a multilayer Sphingomyelin-gel structure upon the sensing surface of the device, we may proceed through the specific protocol regarding experimental measurements described in Fig. 3 and 5 so as to monitor the dynamic evolution of the sphingomyelin lipid phase transition.

\subsection{Photonics detection of Sphingolipid gel/fluid phase transition}

Considering this new experiment, the reference pattern is then related to the wavelength resonant comb which represents the optical quantification relevant with the novel MSM gel upper cladding $300 \mathrm{~nm}$ in thickness. In order to avoid any prospective metastable state of supercooling during the determination of the melting point $\mathrm{T}_{\mathrm{m}}$, all measurements have to be carried out according to increasing 
temperature values, namely from $16{ }^{\circ} \mathrm{C}$ to $42{ }^{\circ} \mathrm{C}$. At first, the TDWS of such an overall resonant structure fitted with an upper-cladding involving a $750 \mathrm{~nm}$ set of MSM multilayers has been evaluated as about $\mathrm{d} \lambda_{\mathrm{i}} / \mathrm{dT}=21.5 \mathrm{pm} /{ }^{\circ} \mathrm{C} \pm 1 \mathrm{pm} /{ }^{\circ} \mathrm{C}$ with an ultra-high stability along the whole set of wavelengths $\lambda_{\mathrm{i}}$ of the family spectra, Fig. 6(a). Then, such a MSM upper cladding seems to strengthen the quasi-athermal property of the global photonic structure already verified in previous experimental measurements. Fig. 6(b) and 6(c) depict the evolution in temperature of the spectrum-parameters FSR, FWHM and then Q-factor and Finesse. To the first order, the FSR remains quasi-constant in our global system as the temperature is increased: such a behavior is certainly linked to the high opto-thermal stability of such DUV210 polymer, but as the FWHM falls off against temperature (T), then the finesse is increased. The fingers of the frequency comb shrink in, somehow at a quasi-constant period, prior stabilization at the melting point characterizing such a phase transition. It is clear that such experimental measurements throw light on the FWHM variation $\Delta(\delta \lambda)_{\mathrm{T}}$ showing off a strong change of behavior with regards to temperature with a highly noticeable inversion of the slope (Fig. 6(b)). These devices and sensors are based on twin 'coupling plus resonance' physics, namely a tunnel effect through a gap plus an optical geometric and cyclic resonance. According to the devices, the equations of the electromagnetism dealing with a "coupler and resonator" set describe and settle the operating regimes which specify the quantified values of the coupling $\mathrm{K}$ (weak or strong) from the guide to the resonator. Indeed, the transmission and the FWHM of such devices are functions of intrinsic parameters such as both $\mathrm{K}$ the coupling factor and A the intra-cavity losses. The equilibrium of the regime is clearly broken by the dynamic of the Sphingomyelin and its own phase transition. According to the evolution of the FWHM (Fig. 6(b)), the regime grows towards a stronger K-coupling (as FWHM is decreased) before levelling off at the exact beginning of the phase transition of Sphingomyelin or $\mathrm{T}_{\mathrm{m}}$ melting temperature. Both Q-factor and finesse being inversely proportional to the FWWM $\delta \lambda$ (which is an arcos function of K, A and insertion losses $\gamma$ ), can be written as a (cst/ $\delta \lambda$ )law due to the low-thermal behavior of the photonic structure: both quantities feature a same slope inversion against temperature as a change occurs between the two gel/fluid states (Fig. 6(c)).

However, it can be seen that the TDWS doesn't change significantly (Fig. 4 (a) and Fig. 6 (a)). An additionally important reason for the Q-factor and Finesse F evolutions measured in Fig. 6 (c) is linked to the change of the optical scattering properties during the phase transition process [40]. The Q-value is directly linked to three optical losses phenomena: scattering, radiation and absorption: $1 / \mathrm{Q}_{\text {total }}=1 / \mathrm{Q}_{\text {Scatt }}+1 / \mathrm{Q}_{\mathrm{Rad}}+1 / \mathrm{Q}_{\mathrm{Absorp}}$ [41]. The scattering loss appears as an important factor in the change of our MR properties at the lipids phase transition due to the variation of the surface asperities and due to our principle of measurement. Hence, the ability to detect the specific gel/fluid transition phase of Sphingomyelin lipids and the efficiency to pinpoint the melting temperature at $\mathrm{T}_{\mathrm{m}}=31 \pm 0.5{ }^{\circ} \mathrm{C}$ have been precisely demonstrated.

\subsection{Differential scanning calorimetry}

We reproduce this experiment in presence of Milli Q water in order to compare the temperature values obtained by way of the differential scanning calorimetry (DSC) conventional technique and those resulting from the photonic sensor detections. It can be noted that we have used the same heating rate respectively for the DSC and the photonics measurements close to a few ${ }^{\circ} \mathrm{C} / \mathrm{min}$. DSC thermogram and light sensor detection were performed on the same batch and in the same solvent. The melting behavior of the sphingomyelin was monitored using differential scanning calorimetry (DSC) (DSC Q20; TA Instruments, Guyancourt, France). $2 \mathrm{mg}$ of dry lipids were loaded into the DSC sample pan and hydrated with Milli Q water to reach the final concentration of $20 \%$ wt sphingomyelin. The 
pan was sealed. An empty, hermetically sealed, aluminum pan was used as reference. The calorimeter was calibrated with indium $\left(5.1 \mathrm{mg}, \Delta \mathrm{H}=31.24 \mathrm{~J} / \mathrm{g}\right.$, melting point $\left.=156.48^{\circ} \mathrm{C}\right)$, assessing the accuracy of the temperature measurement to $+/-0.1^{\circ} \mathrm{C}$ and $+/-10 \%$ for the heat flow. The lipid sample was first heated up to $70^{\circ} \mathrm{C}$ then cooled down to $10^{\circ} \mathrm{C}$ and finally heated up to $70^{\circ} \mathrm{C}$, the Fig.7 (a) presents the second heating thermogram. The thermogram recorded on heating showed a broad endothermic transition (about $15^{\circ} \mathrm{C}$ wide) with a shoulder on the high temperature side between 36 and $40^{\circ} \mathrm{C}$. The melting temperature of sphingomyelin associated to the transition from the gel phase to the fluid phase was determined from the onset point, viz. the intersection between the baseline and the line tangent to the main peak. The transition was found at $\mathrm{T}_{\mathrm{m}}=31^{\circ} \mathrm{C}$ in the heating thermogram recorded at $5{ }^{\circ} \mathrm{C} / \mathrm{min}$. Three scanning rates $\left(0.5,5\right.$ and $\left.10{ }^{\circ} \mathrm{C} / \mathrm{min}\right)$ were used to assess hypothetical kinetic effects on the shape of the thermogram. It confirmed that the $\mathrm{T}_{\mathrm{m}}$ is not affected by the scanning rate, as showed in Fig. 7 (b), and that the shoulder actually reflects thermodynamical features of the phase transition and not kinetic distortions. Finally, the calorimetric results are in accordance with the literature. The lower value of the melting temperature $\mathrm{T}_{\mathrm{m}}=31^{\circ} \mathrm{C}$ with respect to $\mathrm{T}_{\mathrm{m}}=35^{\circ} \mathrm{C}$ obtained in Guyomarc' $h$ et al. can be attributed to differences in the sample composition. Indeed, the presence of ions in the solvent could displace the phase transition by changing the head-group packing which adds lateral strain shifting the $T_{m}$ up to high values. In contrast with pure molecule phase transition, the thermogram showed a broad peak, the phase transition occurs on a temperature range of several degrees. This effect is related to the length heterogeneity of the hydrocarbon chains. Milk sphingomyelins present long chains containing 20, 22, 23, or 24 carbons (Avanti polar web site, https://avantilipids.com/product/860063/). Comparing the $\mathrm{T}_{\mathrm{m}}$ value with sphingomyelin obtained from the other sources such as egg or brain, $\mathrm{T}_{\mathrm{m}}$ of milk sphingomyelin is lower due to the presence of more unsaturation hydrocarbon chains (saturated/unsaturated ratio is $27.33 \%$ ). If we analyze the peak evolution, the exothermic one features its maximum value with $31^{\circ} \mathrm{C}$, corroborating exactly the result stemming from our light-sensor performance. The DSC thermogram and its analysis were described in Fig. 7. The $\mathrm{T}_{\mathrm{m}}$ value measured is $31^{\circ} \mathrm{C}$ corroborating exactly the result stemming from our light-sensor performance.

In comparison, with such previous photonics sensor, we used a single $3 \mu \mathrm{L}$ drop of a solution at 1 $\mathrm{mg} / \mathrm{mL}$, entailing $3 \mu \mathrm{g}$ deposited on the chip which contains the resonators. However, we have to take into account the surface of the micro-resonator (MR) which is the probe, because it comes up as a significant interaction between light/lipids matter. The resonant light contributing to the principle of measurement is only located within the MR. Its active surface is $250 \mu \mathrm{m}^{2}$ (Fig. 1 (a)). Then, for the abovementioned $750 \mathrm{~nm}$-thickness on top of this active-MR surface (with $250 \mu \mathrm{m}^{2}$ being 22000 times less than the initial $5.5 \mathrm{~mm}^{2}$ ), the quantity of MSM lipids to be considered as interacting with the light is then: $1.07 \times 10^{11}$ molecules or $1.78 \times 10^{-13} \mathrm{~mol}$ or $1.426 \times 10^{-10} \mathrm{~g}$. Then, when compared with the $2 \mathrm{mg}$ quantity of lipids required with the DSC method, we have used quite less lipids (with a $1.4 \times 10^{7}$ factor) in interaction with the light in such a device and principle of measurement.

\section{Conclusion}

This transverse work features key points distributed over several levels considering: the nature of the specific organic with regard to materials, the technologies of thin layer processes for shaping the chips, associated with the method of deposition in biology (called fusion vesicle deposition method) and the whole protocol of measurements developed for such sensors.

In conclusion, we have experimentally demonstrated, with such a resonant light principle, the interest of a new approach to achieve milk Sphingomyelin (MSM) phase transition detection: indeed, a significantly reduced amount of material is required (fourteen millions times less), when compared with the conventional method, the so-called differential scanning calorimetry (DSC). The temperature 
melting point determination is also presented into our work as regards such a first order transition measurement. This approach is most inviting considering the low cost development fabrication of racetrack polymer photonic micro-resonators. To this end, we have presented in details both the design and a straightforward procedure based on deep UV technology addressing the specific UV210 polymer so as to obtain adequate low thermal resonance mechanisms, without resorting at any time to expensive electron beam lithography technologies. The temperature dependent wavelength shift $(\mathrm{d} \lambda / \mathrm{dT})$ and the $\mathrm{TO}$ coefficient $\left|\mathrm{dn}_{\text {eff }}^{\text {syst }} / \mathrm{dT}\right|$ characterizing the quantified resonances and optogeometric properties of the UV210-device have been measured: such quantities represent somehow the sensitivity of the eigenvalues $\mathrm{n}_{\mathrm{eff}}^{\text {syst }}$ of the photonic system against temperature and both have been evaluated with values enough low for such SPH phase transition detections and applications. The overall experimental protocol related to the appropriate vesicle lipid deposition biology process plus original statistical operation treatments of the family spectra have clearly demonstrated the relevance of the approach to quantifying the point of gel/fluid sphingomyelin lipid phase transition. Furthermore, such UV210 structures based on resonance functionalities associated to our protocol of measurements may also be applicable to investigate other families of sphingolipid in biology (or mixes of them), that present various lengths of hydrophobic tails (carbon chains): here our approach comes up as most appropriate to investigate the shift of the relevant melting point phase transition in such cases of lipid mixtures.

\section{References}

[1] K.J. Vahala, Optical microcavities; advanced series in applied physics, World Scientific Publishing, 2004.

[2] D.G. Rabus, Integrated ring resonators The Compendium, Springer Verlag: Berlin Heidelberg New-York, 2007.

[3] T.M. Benson, S.V. Boriskina, P. Sewell, A. Vukovic, S.C. Greedy, A.I. Nosich, Micro-optical resonators for microlasers and integrated optoelectronics, Springer Netherlands, 2005.

[4] F. Qiu, A.M. Spring, H. Miura, D. Maeda, M.A. Ozawa, K. Odoi, S. Yokoyama, Athermal hybrid silicon/polymer ring resonator electro-optic modulator, ACS Photonics 3 (2016) 780783.

[5] B. Bêche, A. Potel, J. Barbe, V. Vié, J. Zyss, C. Godet, N. Huby, D. Pluchon, E. Gaviot, Resonant coupling into hybrid 3D micro-resonator devices on organic/biomolecular film/glass photonic structures, Opt. Comm. 283 (2010) 164-168.

[6] R. Fenollosa, F. Ramiro-Manzano, M. Tymczenko, F. Meseguer, Porous silicon microspheres : synthesis, characterization and application to photonic microcavities, RSC J. Mater. Chem. 20 (2010) 5210-5214.

[7] N. Huby, D. Pluchon, N. Coulon, M. Belloul, A. Moreac, E. Gaviot, P. Panizza, B. Bêche, Design of organic 3D microresonators with microfluidics coupled thin-film processes for photonic applications, Opt. Comm. 283 (2010) 2451-2456.

[8] N. Gaber, M. Malak, X. Yuan, K.N. Nguyen, P. Basset, E. Richalot, D. Angelescu, T. Bourouina, On the free-space gaussian beam coupling to droplet optical resonators, RSC Lab Chip 13 (2013) 826-833.

[9] R. Castro-Beltran, N. Huby, G. Loas, H. Lhermite, D. Pluchon, B. Bêche, Improvement of efficient coupling and optical resonances by using taper-waveguides couples to cascade of UV210 polymer micro-resonators, J. Micromech. Microeng. 24 (2014) 125006.1-125006.7. 
[10] H. Ma, K.Y. Jen, L.R. Dalton, Polymer-based optical waveguides: materials, processing, and devices, Adv. Mater. 14 (2002) 1339-1365.

[11] Z. Zhang, P. Zhao, P. Lin, F. Sun, Thermo-optic coefficients of polymers for optical waveguide applications, Polymer 47 (2006) 4893-4896.

[12] J. Scheuer, A. Yariv, Fabrication and characterization of low-loss polymeric waveguides and micro-resonators, J. Eur. Opt. Soc. 1 (2006) 06007.1-06007-5.

[13] M. Lebental, J.S. Lauret, R. Hierle, J. Zyss, Highly directional stadium-shaped polymer microlasers, Appl. Phys. Lett. 88 (2006) 031108.1-031108.1-3.

[14] K. Takazawa, J.I. Inoue, K. Mitsuishi, Optical microring resonators constructed from organic dye nanofibers and their application to miniaturized channel drop/add filters, ACS Appl. Mater. Interfaces 5 (2013) 6182-6188.

[15] A.A.P. Trichet, J. Foster, N.E. Omori, D. James, P.R. Dolan, G.M. Hughes, C. Vallance, J.M. Smith, Open-access optical microcavities for lab-on-chip refractive index sensing, RSC Lab Chip 14 (2014) 4244-4249.

[16] S.I. Shopova, I.M. White, Y. Sun, H. Zhu, X. Fan, G. Frye-Mason, A. Thompson, S.J. Ja, Oncolumn micro gaz chromatography detection with capillary-based optical ring resonators, ACS Anal. Chem. 80 (2008) 2232-2238.

[17] M.T. Marty, C.D. Kuhnline Sloan, R.C. Bailey, S.G. Sligar, Nonlinear analyte concentration gradients for one-step kinetic analysis employing optical microring resonators, ACS Anal. Chem. 84 (2012) 225556-5564.

[18] R. Castro-Beltran, N. Huby, V. Vié, H. Lhermite, L. Camberlein, E. Gaviot, B. Bêche, A laterally coupled UV210 polymer racetrack micro-resonator for thermal tunability and glucose sensing capability, Adv. Device Mater. 1 (2015) 80-87.

[19] R. Ahmed, A.A. Rifat, A.K. Yetisen, M.S. Salem, S.H. Yun, H. Butt, Optical microring resonator based corrosion sensing, RSC Adv. 6 (2016) 56127-56133.

[20] J. Topolanick, F. Vollmer, Photoinduced transformations in bacteriorhodopsin membrane monitored with optical microcavities, Biophys. J. 92 (2007) 2223-2229.

[21] H. Zhu, I.M. White, J.D. Suter, M. Zourob, X. Fan, Opto-fluidic micro-ring resonators for sensitive label-free viral detection, RSC Analyst 133 (2008) 356-360.

[22] H.K. Hunt, A.M. Armani, Label-free biological and chemical sensors, RCS Nanoscale 2 (2010)1544-1559.

[23] M. Calleja, P.M. Kosaka, A. San Paulo, J. Tamayo, Challenges for nanomechanical sensors in biological detection, RSC Nanoscale 4 (2012) 4925-4938.

[24] C. Ciminelli, C.M. Campanella, F. Dell'Olio, C.E. Campanella, M.N. Armenise, Label-free optical resonant sensors for biochemical applications,Progress Quantum. Electron. 37 (2013) 51-107.

[25] M.N.V. Passaro, B. Troia, M. La Notte, F. De Leonardis, Photonic resonant microcavities for chemical and biochemical sensing, RSC Adv. 3 (2013) 25-44.

[26] D.C. Kim, K.P. Armendariz, R.C. Dunn, Integration of microsphere resonators with bioassay fluidics for whispering gallery mode imaging, RSC Analyst 138 (2013) 3189-3195.

[27] J. Su, Label-free single exosome detection using frequency-locked microtoroïd optical resonators, ACS Photonics 2 (2015) 1241-1245.

[28] I.A. Grimaldi, G. Testa, R. Bernini, Flow through ring resonator sensing platform, RSC Adv. 5 (2015) 70156-70162.

[29] S.W. Chiu, S. Vasudevan, E. Jakobsson, R.J. Mashl, H.L. Scott, Structure of sphingomyelin bilayers : a simulation study, Biophys. J. 85 (2003) 3624-3635.

[30] K.P. Shaw, N.J. Brooks, J.A. Clarke, O. Ces, J.M. Seddon, R.V. Law, Pressure-temperature phase behavior of natural sphingomyelin extracts, RSC Soft Matter 8 (2012) 1070-1078. 
[31] F. Guyomarc'h, S. Zou, M. Chen, P.E. Milhiet, C. Godefroy, V. Vié, C. Lopez, Milk sphingomyelin domains in biomimetic membranes and the role of cholesterol: morphology and nanomechanical properties investigated using AFM and force spectroscopy, ACS Langmuir 330 (2014) 6516-6524.

[32] H.M.G. Barriga, E.S. Parsons, N.L.C. McCarthy, O. Ces, J.M. Seddon, R.V. Law, N.J. Brooks, Pressure-temperature phase behavior of mixtures of natural sphingomyelin and ceramide extracts, ACS Langmuir 31 (2015) 3678-3686.

[33] N.B. Colthup, L.H. Daly, S.E. Wiberley, Introduction to infrared and Raman spectroscopy, advanced series in applied physics, Academic Press, INC, Boston New-York London Sydney, 1990.

[34] F. Qiu, F. Yu, A.M. Spring, S. Yokoyama, Athermal silicon nitride ring resonator by photobleaching of disperse red 1-doped poly(methyl methacrylate) polymer, Opt. Lett. 37 (21012) 4086-4088

[35] G. Qian, T. Zhang, L-J. Zhang, J. Tang, X-Y. Zhang, Y. Lu, F-H. Wan, Demonstrations of centimeter-sacle polymer resonator for resonant integrated optical gyroscope, 237 (2016) 29-34.

[36] N. Razali, R. Mohamed, A.A. Ehsan, C.S. Kuang, S. Shaari, Thermo-optic coefficient of different photosensitive acrylate polymers for optical application, J. Nonlinear Opt. Phys. Mat. 14 (2005) 195-201.

[37] Li, X.; Cao, Z-Q.; Shen, Q-S.; Meng, Q-H., Huang, D-Y.; Guo, K-P.; Qiu, L.; Shen, Y-Q. Anisotropy in thermo-optic coefficient of different polymer systems by attenuated total reflection configuration. Chin. Phys. Lett. 2006, 23, 998-1001.

[38] Zhang, Z.; Zhao, P.; Lin, P.; Sun, F. Thermo-optic coefficients of polymers for optical waveguide applications. Polymer Comm. 2006, 47, 4893-4896.

[39] D.A. Brown, E. London, Functions of lipid rafts in biological membranes., Annu.Rev. Cell Dev. Biol. 14 (1998) 111-136.

[40] N. Michel, A-S. Fabiano, A. Polidori, R. Jack, B. Pucci, Determination of phase transition temperatures of lipids by light scattering, Chem. Phys. Lipids 139 (2006) 11-19.

[41] D. Pluchon, N. Huby, V. Vié, P. Panizza, B. Bêche, AFM analysis on polymer optical microresonators: investigation on quality factor origin, Opt. Photon. J. 3 (2013) 291-295. 


\section{Figure Captions}

Fig. 1. Quality control of the photonic device including waveguide coupled with resonator, optogeometric characterizations and measurements of parameters. (a) Top view photograph (x 40) of the racetrack polymer resonator, $\mathrm{R}=\mathrm{L}_{\mathrm{c}}=15 \mu \mathrm{m}, \mathrm{w}=2 \mu \mathrm{m}$, with parameters $\mathrm{R}, \mathrm{L}_{\mathrm{c}}$ and $\mathrm{w}$ the radius, the coupling length and the width respectively. (b) AFM imaging, $h=860 \mathrm{~nm}, \mathrm{~g}=540 \mathrm{~nm}$, with $\mathrm{h}$ the height and $\mathrm{g}$ the gap; the roughness polymer surface has been measured $<2 \mathrm{~nm}$. (c), (d), (e), (f) Raman imaging and spectroscopy. (c),(d) Cross-sectional Raman imaging of the $\mathrm{Si}_{\mathrm{SiO}} / \mathrm{UV} 210$ and Raman spectroscopy analyses of respective materials. (e) Raman top view imaging of the overall photonic device@ $1002 \mathrm{~cm}^{-1}$, UV210 polymer line. (f) Raman profile trace of the gap coupling (g ranging around $550 \mathrm{~nm}$ ), with @ $521 \mathrm{~cm}^{-1}$ and Si first order line.

Fig. 2. Sphingomyelin. (a) Chemical structure and symbolic representation of a 16:0 sphingomyelinmolecule showing both the hydrophobic tails and hydrophilic head-group. (b) Schematic diagram of the fusion vesicle deposition method applied to the photonic device so as to achieve the milk sphingomyelin (MSM) multilayers in gel configuration.

Fig. 3. (a) Synoptic of the experimental protocol (with and without lipids). (b) MBP (Newport) top view imaging of the photonic device under test assessing both light propagation and coupling from the waveguide to the resonators plus the apt circulation into the racetrack loop; inset, cross-sectional detection and imaging of the exit $\mathrm{TE}_{00}$-singlemode. (c) Typical resonant spectrum at fixed temperature of the photonic device detected and stored by OSA and computer; $\lambda_{\mathrm{i}}$ (i, integer), $\Delta \lambda$ and $\delta \lambda$ parameters respectively associated with the resonant wavelengths, the FSR and the FWHM; inset FFT calculus of the comb-shaped periodic wavelength allowing relevant FSR measurement.

Fig. 4. (a) Thermal behavior of the photonic structure $\mathrm{Si} / \mathrm{SiO}_{2} / \mathrm{UV} 210 /$ air (without lipids) yielded from a statistical analysis involving 20 resonant spectra with increased temperature ( $\mathrm{T}$ ranging [16$\left.38]^{\circ} \mathrm{C}\right) ;\left(\mathrm{d} \lambda_{\mathrm{l}} / \mathrm{dT}\right) \approx 28 \mathrm{pm} /{ }^{\circ} \mathrm{C}$. (b) Quasi-invariance in temperature of the FSR and the FWHM of the optical spectra considering related $\lambda_{\mathrm{i}}$ resonant peaks. (c) Evolution in temperature of the Q-factor and Finesse of the photonic device without lipids.

Fig. 5. Global photonics platform and lipid 'gel/fluid' phase transition detection, thermal and mechanical systems controls, computing and signal processing. Outline of the photonics characterization platform used for the optical resonance (in visible wavelength) into the MRs and the lipid gel/liquid phase transition detection: Superlum red broadband source (SLD $331 \mathrm{HP} 3, \lambda_{0}=795 \mathrm{~nm}$, $\Delta \lambda=40 \mathrm{~nm}$ plus single mode fiber (SMF)), nanopiezoelectric positioners/manipulators (PI E-563I.3), optical imaging (PulNix CCD camera) and optical spectral analysis (OSA Ando AQ-6315E and Anritsu MS9710C).

Fig. 6. Detection of the gel-liquid phase transition of MSM lipids. (a) Low-thermal behavior of the photonic structure $\mathrm{Si} / \mathrm{SiO}_{2} / \mathrm{UV} 210 / \mathrm{MSM}$ yielded from a statistical analysis involving 20 resonant spectra with increased temperature $\left(\mathrm{T}\right.$ ranging $\left.[16-42]^{\circ} \mathrm{C}\right) ;\left(\mathrm{d} \lambda_{\mathrm{l}} / \mathrm{dT}\right) \approx 21 \mathrm{pm} /{ }^{\circ} \mathrm{C}$. (b) Evolution in temperature ( $\mathrm{T}$ ranging $[16-42]^{\circ} \mathrm{C}$ ) of the FSR and the FWHM of the photonic structures $\mathrm{Si} / \mathrm{SiO}_{2} / \mathrm{UV} 210 / \mathrm{MSM}$ lipids/air: highlighting clear breaks of slopes as a way to assess the melting temperature $\mathrm{T}_{\mathrm{m}}$ of MSM lipids with $\mathrm{T}_{\mathrm{m}} \approx[31-32]^{\circ} \mathrm{C}$. (c) Evolution in temperature of the Q-factor and Finesse of the photonic device under MSM test, determination of $\mathrm{T}_{\mathrm{m}} \approx[31-32]^{\circ} \mathrm{C}$.

Fig. 7. DSC experiments on milk sphingomyelin, (a) thermogram (endothermic heat flow up) and determination of the $T_{m}$. (b) $T_{m}$ extracted from the thermograms recorded at different scanning rate. 
Fig. 1

(a)

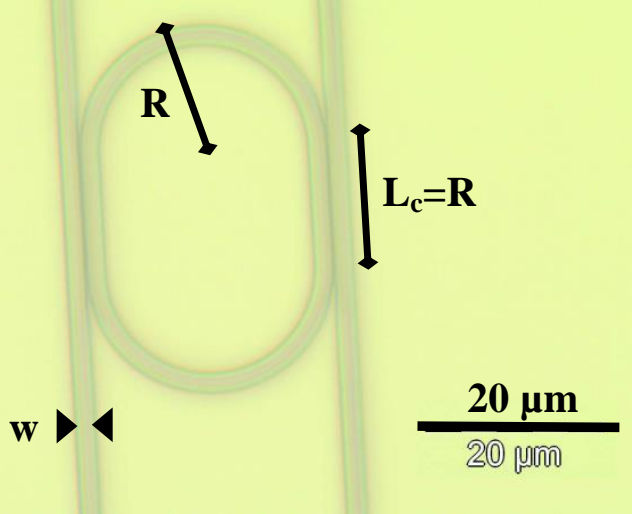

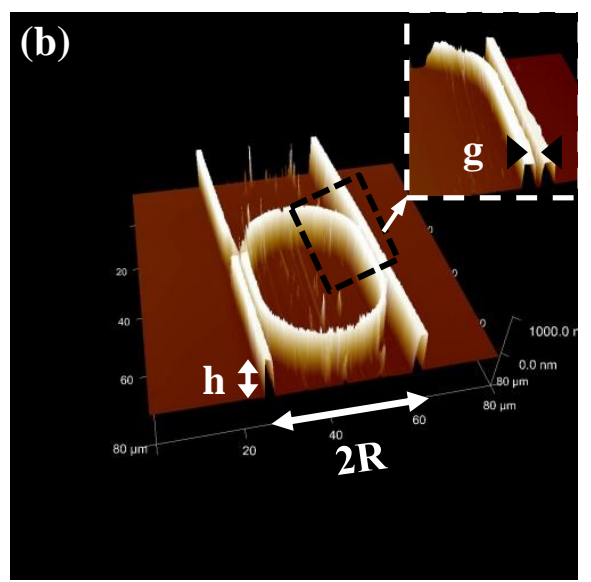
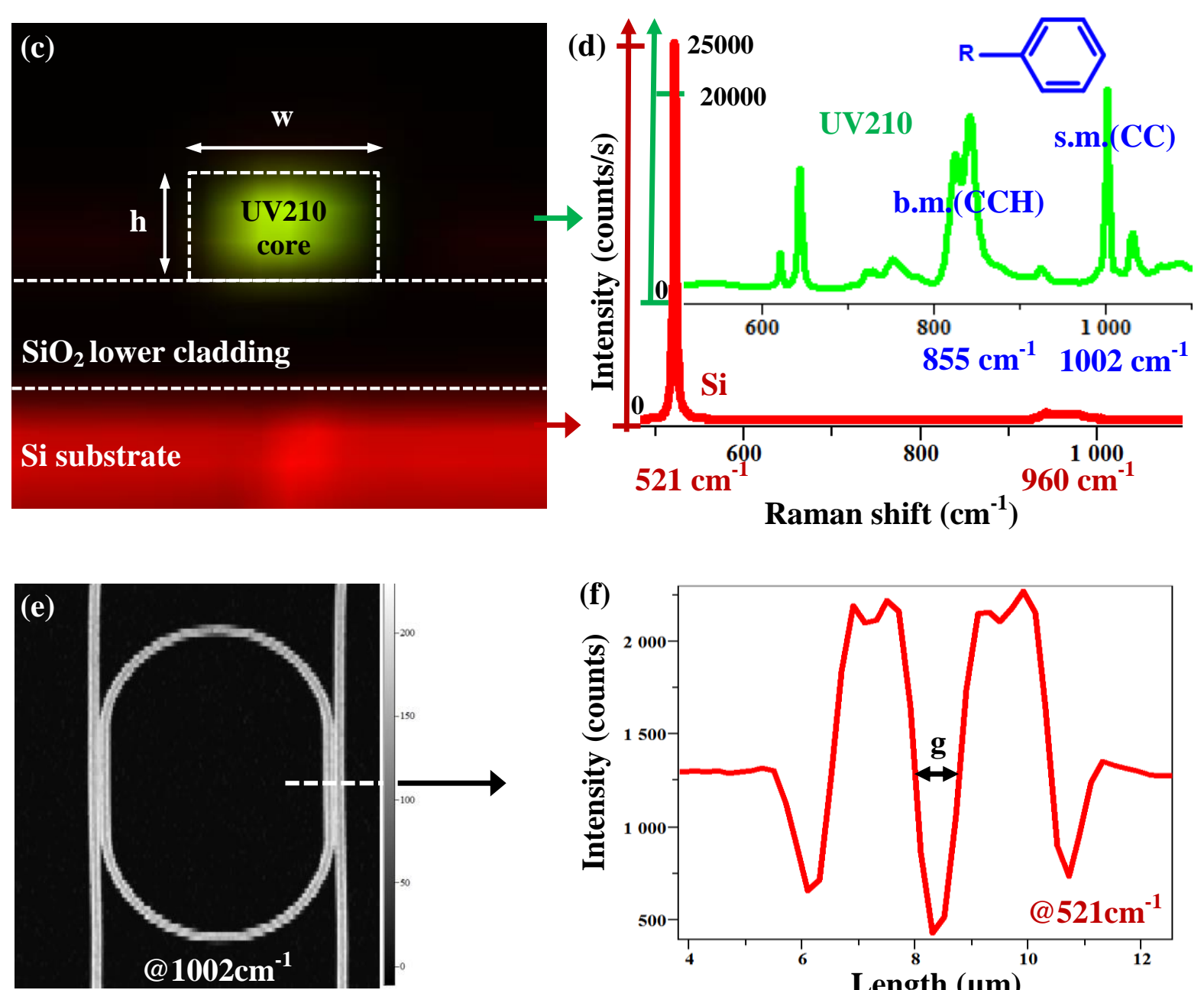
Fig. 2
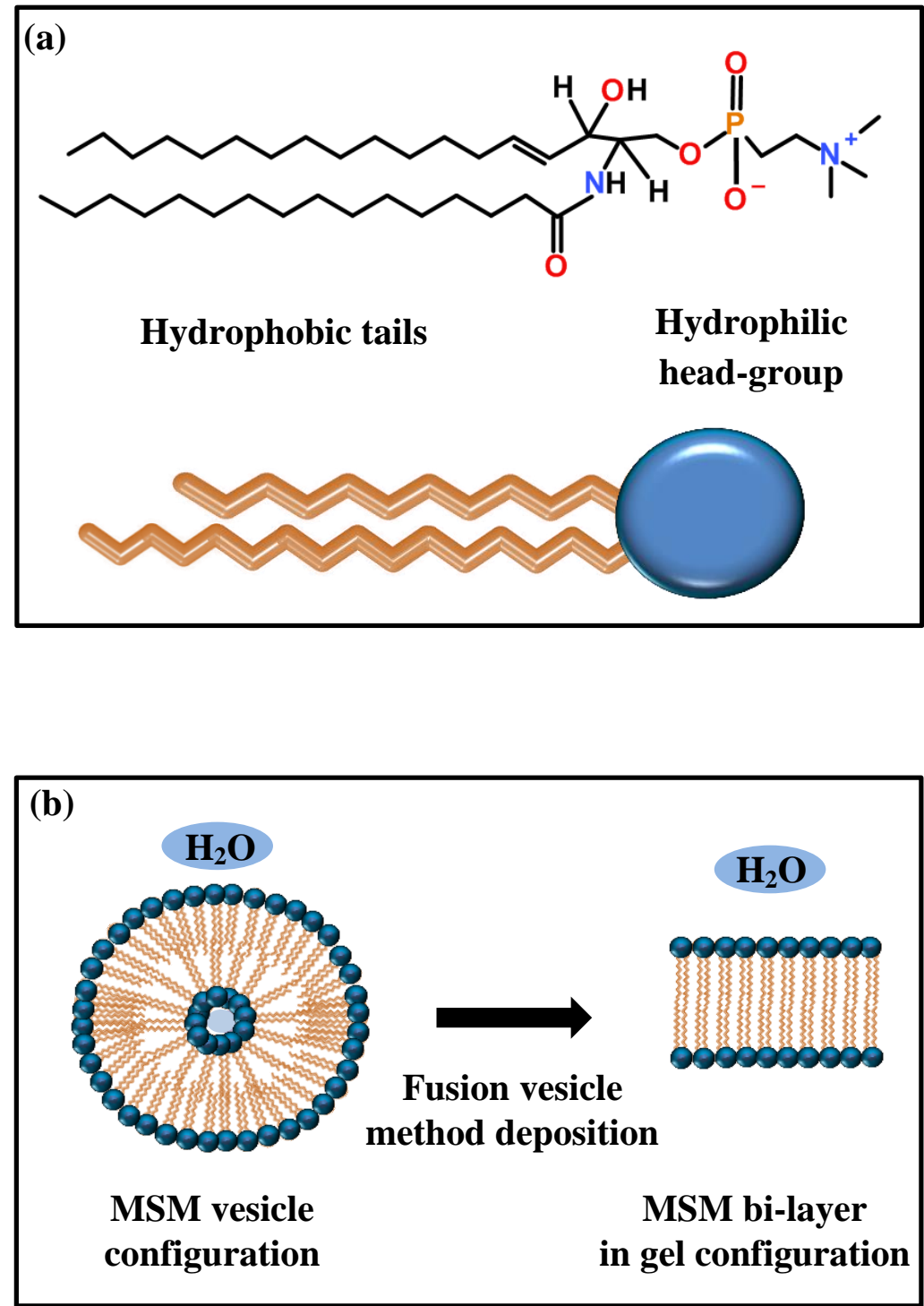
Fig. 3

\begin{tabular}{|c|c|c|}
\hline \multicolumn{3}{|c|}{$\begin{array}{l}\text { (a) Synoptic of the method - experimental protocol - principles } \\
\text { (with and without lipids) }\end{array}$} \\
\hline Optical modes preparation & Optical injection - tests & $\begin{array}{l}\text { Optical spectral measurements } \\
\text { Statistical analyses }\end{array}$ \\
\hline $\begin{array}{l}\text { - Broadband laser source } \\
\left(\lambda_{0}=795 \mathrm{~nm}, \mathrm{FWHM}=40 \mathrm{~nm}\right) \\
\text { - Singlemode fiber } \\
\text { - Polarizer }(\text { TE-TM })\end{array}$ & $\begin{array}{l}\text { - Nano-positioners } \\
\text { - Thermal control of the } \\
\text { devices } \\
\text { - Powermeter } \\
\text { - Optical imaging, CCD- } \\
\text { camera, video (upper + } \\
\text { cross sectional view, see } \\
\text { (b)) }\end{array}$ & $\begin{array}{l}\text {-Spectral analyses }(\text { see }(\mathrm{c}))+ \\
\text { FFT calculus } \\
\text { - Successive dynamic } \\
\text { measurements } \\
\text { - Computer and statistical } \\
\text { treatments of the data }\end{array}$ \\
\hline
\end{tabular}
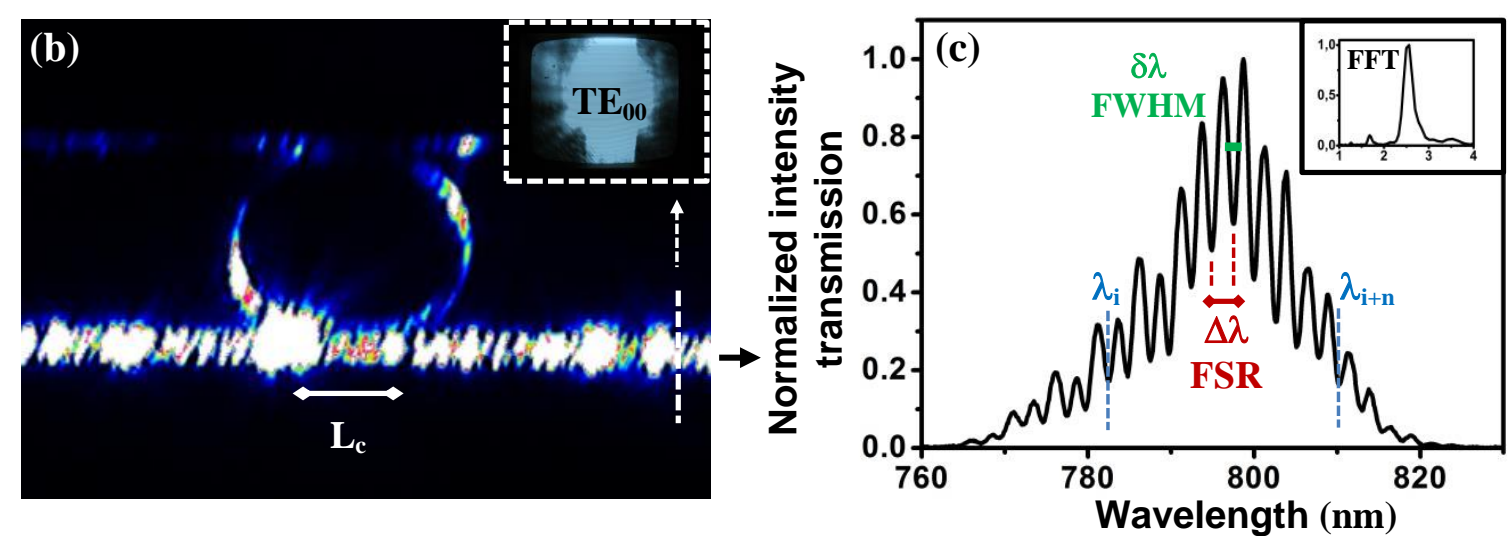
Fig. 4
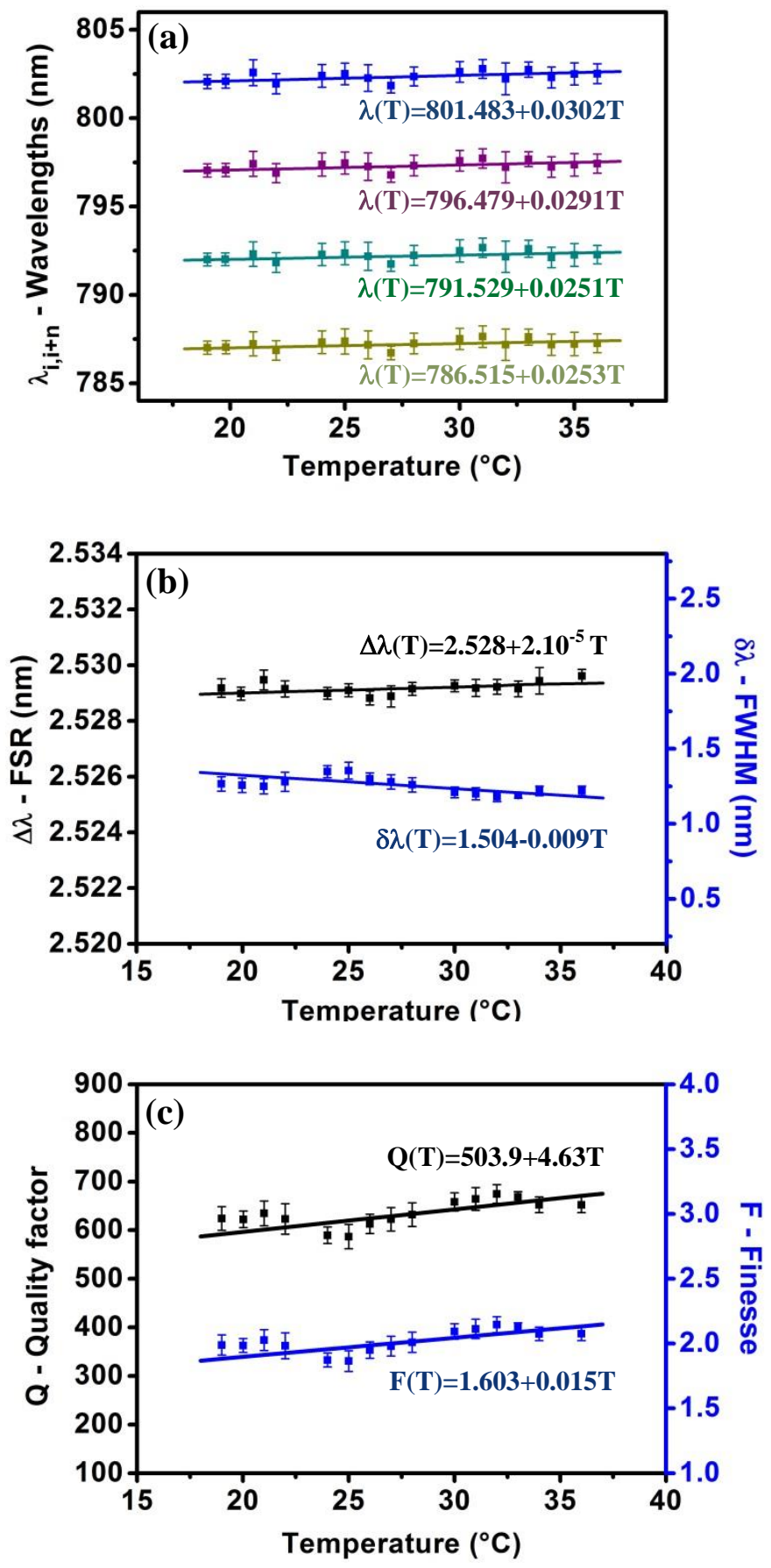
Fig. 5

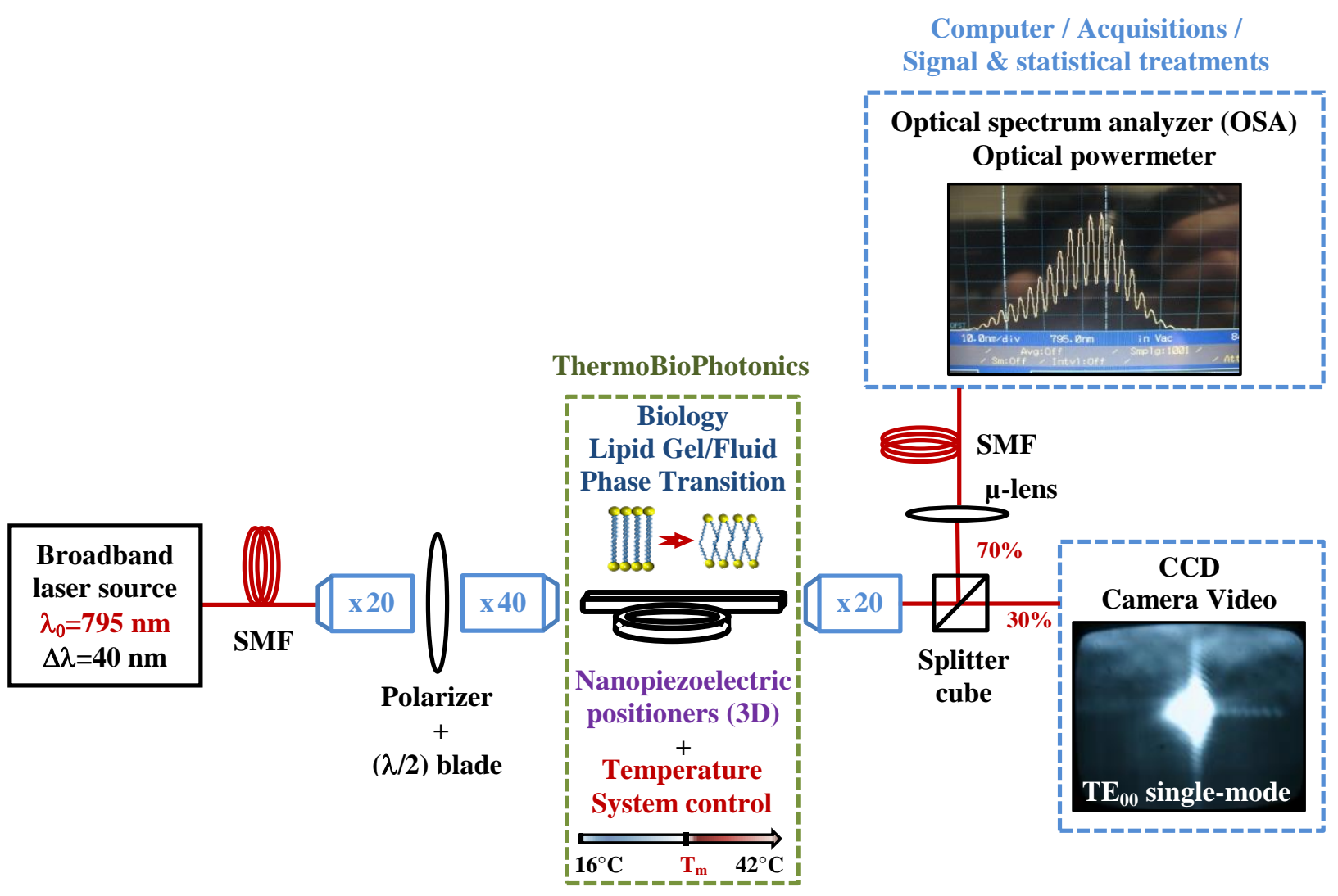


Fig. 6
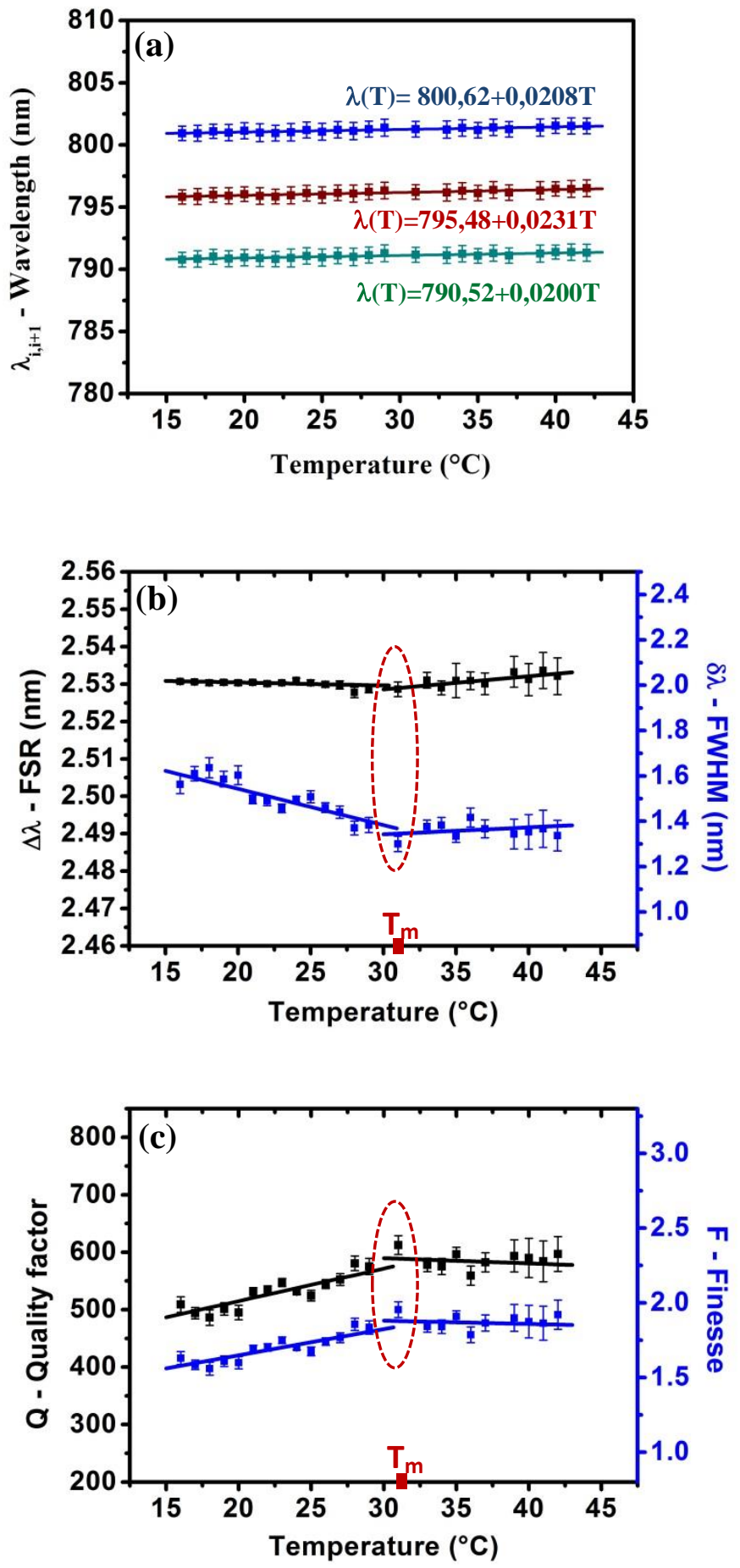
Fig. 7
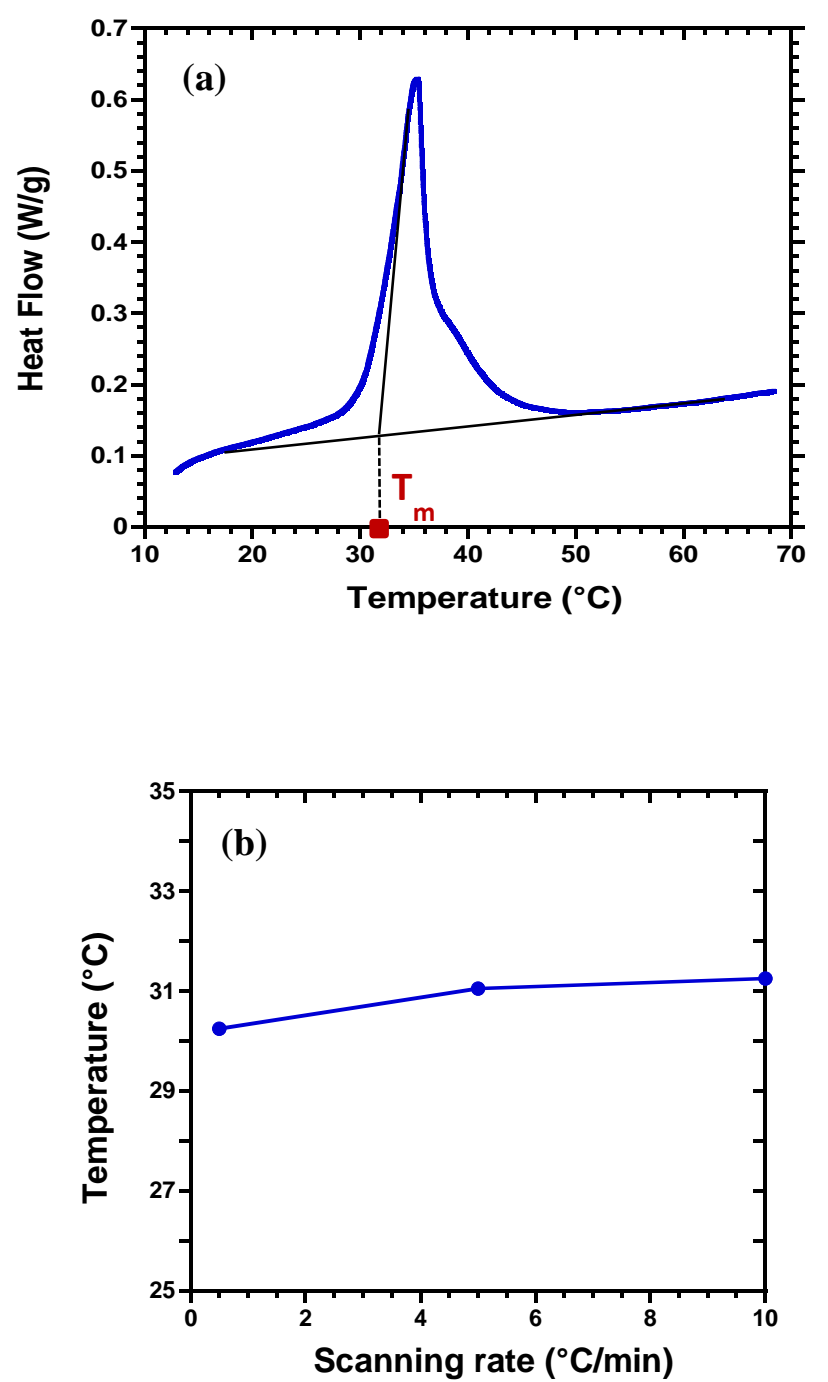


\section{Table Caption}

Table 1. Cut-thickness values for $\mathrm{TE}$ and $\mathrm{TM}(\mathrm{m}=0$ and 1$) ; \mathrm{nSiO}_{2}=1.453$ and $\mathrm{n}_{\mathrm{DUv} 210}=1.595$ at $\lambda_{0}=795 \mathrm{~nm}$.

Table 2. Serial of thin layers processes steps in cleanroom for deep UV210 polymer MRs ; (v, speed; a, acceleration; $t$, time; $\mathrm{T}$, temperature; E, exposure dose). 
Table 1

\begin{tabular}{lccc}
\hline & & TE-polarization & TM-polarization \\
\hline \multirow{2}{*}{$\mathbf{h}_{\mathbf{c}}(\mathbf{n m})$} & $(\mathrm{m}=0)$ & 195 & 256 \\
\cline { 2 - 4 } & $(\mathrm{m}=1)$ & 800 & 860 \\
\hline
\end{tabular}


Table 2

\begin{tabular}{cc}
\hline $\begin{array}{c}\text { Steps and Procedures } \\
\text { UV210 polymer }\end{array}$ & Parameters \\
\hline $\begin{array}{c}\text { Spin-coating }(\mathrm{v}, \mathrm{a}, \mathrm{t},) \\
\text { thickness, roughness }\end{array}$ & $\begin{array}{c}900 \mathrm{rpm}, 5000 \mathrm{rpm} / \mathrm{s}, 30 \mathrm{~s} \\
\sim 800-850 \mathrm{~nm},<3 \mathrm{~nm}\end{array}$ \\
\hline Softbake $(\mathrm{t}, \mathrm{T})$ & $3 \mathrm{~min}$ at $140^{\circ} \mathrm{C}$ \\
\hline $\begin{array}{c}\text { Deep UV }(\lambda=248 \mathrm{~nm}) \text { exposure } \\
\text { (OSRAM Hg lamp, dose, } \mathrm{t})\end{array}$ & $\mathrm{E}=20 \mathrm{~mJ} / \mathrm{cm}^{2}, 27 \mathrm{~s}$ \\
\hline Post-exposure softbake $(\mathrm{t}, \mathrm{T})$ & $1 \mathrm{~min}$ at $120^{\circ} \mathrm{C}$ \\
\hline Development $(\mathrm{t}$, product $)$ & $30 \mathrm{~s}$, Microposit $\mathrm{MF} \mathrm{CD}-26$ \\
\hline Final softbake $(\mathrm{t}, \mathrm{T})$ & $12 \mathrm{~h}$ to $24 \mathrm{~h}$ at $120^{\circ} \mathrm{C}$ \\
\hline
\end{tabular}

